\title{
On Eccentric Digraphs of Graphs
}

\author{
Medha Itagi Huilgol", Syed Asif Ulla S., Sunilchandra A. R. \\ Department of Mathematics, Bangalore University, Bangalore, India \\ E-mail:medha@bub.ernet.in \\ Received March 11, 2011; revised April 9, 2011; accepted April 12, 2011
}

\begin{abstract}
The eccentricity $e(u)$ of a vertex $u$ is the maximum distance of $u$ to any other vertex of $G$. A vertex $v$ is an eccentric vertex of vertex $u$ if the distance from $u$ to $v$ is equal to $e(u)$. The eccentric digraph $E D(G)$ of a graph (digraph) $G$ is the digraph that has the same vertex as $G$ and an arc from $u$ to $v$ exists in $E D(G)$ if and only if $v$ is an eccentric vertex of $u$ in $G$. In this paper, we have considered an open problem. Partly we have characterized graphs with specified maximum degree such that $E D(G)=G$.
\end{abstract}

Keywords: Eccentric Vertex, Eccentric Degree, Eccentric Digraph, Degree Sequence, Eccentric Degree Sequence

\section{Introduction}

A directed graph or digraph $G$ consists of a finite nonempty set $V(G)$ called vertex set with vertices and edge set $E(G)$ of ordered pairs of vertices called arcs; that is $E(G)$ represents a binary relation on $V(G)$. Throughout this paper, a graph is a symmetric digraph; that is, a digraph $G$ such that $(u, v) \in E(G)$ implies $(v, u) \in E(G)$. If $(u, v)$ is an arc, it is said that $u$ is adjacent to $v$ and also that $v$ is adjacent from $u$. The set of vertices which are from (to) a given vertex $v$ is denoted by $N^{+}(u)\left[N^{-}(u)\right]$ and its cardinality is the out-degree of $v$ [in-degree of $v$ ]. A walk of length $k$ from a vertex $u$ to a vertex $v$ in $G$ is a sequence of vertices

$u=u_{0}, u_{1}, u_{2}, \cdots, u_{k-1}, u_{k}=v$ such that each pair $\left(u_{i-1}, u_{i}\right)$ is an arc of $G$. A digraph $G$ is strongly connected if there is a $u$ to $v$ walk for any pair of vertices $u$ and $v$ of $G$. The distance $d(u, v)$ from $u$ to $v$ is the length of a shortest $u$ to $v$ walk. The eccentricity e $(v)$ of $v$ is the distance to a farthest vertex from $v$. If

$\operatorname{dist}(u, v)=e(u)(v \neq u)$ we say that $v$ is an eccentric vertex of $u$. We define $\operatorname{dist}(u, v)=\infty$ whenever there is no path joining the vertices $u$ and $v$. The $\operatorname{radius} \operatorname{rad}(G)$ and diameter $\operatorname{diam}(G)$ are minimum and maximum eccentricities, respectively. As in [2], the sequential join $G_{1}+G_{2}+G_{3}+\cdots+G_{k}$ of graphs $G_{1}, G_{2}, \cdots, G_{k}$ is the graph formed by taking one copy of each of the graphs

*Part of this paper [1] was presented at the International Conference on Emerging Trends in Mathematics and Computer Applications, MEPCO Schlenk Engineering College, Sivakasi, India (Dec. 2010) and had appeared in the Proceedings of the same.
$G_{1}, G_{2}, \cdots, G_{k}$ and adding in additional edges from each vertex of $G_{i}$ to each vertex in $G_{i+1}$, for $1 \leq i \leq k-1$. Throughout this paper, ' $G=H^{\prime}$ means $G$ and $H$ are isomorphic. The reader is referred to Buckley and Harary [2] and Chartrand and Lesniak [3] for additional, undefined terms.

Buckley [4] defines the eccentric digraph $E D(G)$ of a graph $G$ as having the same vertex set as $G$ and there is an arc from $u$ to $v$ if $v$ is an eccentric vertex of $u$. The paper [4] presents the eccentric digraphs of many classes of graphs including complete graphs, complete bipartite graphs, antipodal graphs and cycles and gives various interesting general structural properties of eccentric digraphs of graphs. The antipodal digraph of a digraph $G$ denoted by $A(G)$, has the vertex set as $G$ with an arc from vertex $v$ in $A(G)$ if and only if $v$ is an antipodal vertex of $u$ in $G$; that is $\operatorname{dist}(u, v)=\operatorname{diam}(G)$. This notion of antipodal digraph of a digraph was introduced by Johns and Sleno [5] as an extension of the definition of the antipodal graph of a graph given by Aravamudhan and Rajendran [6]. It is clear that $A(G)$ is a subgraph of $E D(G)$, and $A(G)=E D(G)$ if and only if $G$ is self centered.

In [7] Akiyama et al. have defined eccentric graph of a graph $G$, denoted by $G_{e}$, has the same set of vertices as $G$ with two vertices $u$ and $v$ being adjacent in $G_{e}$ if and only if either $v$ is an eccentric vertex of $u$ in $G$ or $u$ is an eccentric vertex of $v$ in $G$, that is

$\operatorname{dist}_{G}(u, v)=\min \left\{e_{G}(u), e_{G}(v)\right\}$. Note that $G_{e}$ is the underlying graph of $E D(G)$.

In [8] Boland and Miller introduced the concept of the 
eccentric digraph of a digraph. In [9] Gimbert et al. have proved that $G_{e}=E D(G)$ if and only if $G$ is self-centered. In the same paper, the authors have characterized eccentric digraphs in terms of complement of the reduction of $G$, denoted by $\overline{G^{-}}$Given a digraph $G$ of order $n$, a reduction of $G$, denoted by $G^{-}$, is derived from $G$ by removing all its arcs incident from vertices with outdegree $n-1$. Note that $E D(G)$ is a subgraph of $\overline{G^{-}}$.

In [9], Gimbert et al. have studied on the behaviour of sequences of iterated eccentric digraphs. Given a positive integer $k \geq 2$, the $k^{\text {th }}$ iterated eccentric digraph of $G$ is written as $E D^{k}(G)=E D\left(E D^{k-1}(G)\right)$, where $E D^{0}(G)=G$ and $E D^{1}(G)=E D(G)$. The iterated sequence of eccentric digraphs concerns with the smallest integer numbers $\dot{p}>0$ and $t \geq 0$ such that

$E D^{t}(G)=E D^{p+t}(G)$. We call $p$ the period of $G$ and $t$ the tail of $G$; these quantities are denoted $p(G)$ and $t(G)$ respectively. In $[8,10]$ Boland et al. have discussed many interesting results about eccentric digraphs. Also they have listed open problems about these graphs. One of these open problems is being discussed mainly in this paper. We have characterized graphs with specified maximum degree such that $E D(G)=G$.

\section{Basic Results}

In this section we list some results which are quite evident for eccentric digraphs of graphs.

Remark 1. Since every vertex in a graph has at least one vertex at eccentric distance it follows that every vertex in an eccentric digraph will have out degree at least one.

Remark 2. There exists no directed cycle in an eccentric digraph.

Let $C$ be a directed cycle with edge $u v$ being directed from $u \rightarrow v$ as shown below in Figure 1.

The other edges can be bidirectional. If all the other edges except $u \rightarrow v$ are bidirectional then a symmetric edge $v y_{1}$ indicates the equality of eccentric values of $v$ and $y_{1}$. Likewise

$\operatorname{ecc}\left(y_{1}\right)=e \operatorname{ecc}\left(y_{2}\right)=\cdots=e c c\left(y_{n}\right)=e c c(x)$. Also edge $x u$ is symmetric. Hence, ecc $(u)=\operatorname{ecc}(x)$. So also, $\operatorname{ecc}(v)=\operatorname{ecc}\left(y_{1}\right)=\operatorname{ecc}(x)=e c c(u)$. This contradicts the existence of the directed arc $u \rightarrow v$ as $u$ being tail has less eccentricity as compared to that of $v$.

The same argument can be extended to a directed cycle with more than one directed arc, as the eccentricities go on increasing in the same direction.

The above two conditions are not sufficient for a graph to be an eccentric digraph.

For example consider a symmetric cycle having a pendant vertex adjacent to one of the vertices on the cycle. The pendant vertex having in-degree zero and out-degree one as in Figure 2.

Vertex $x_{i}$ is at eccentric distance from $u$. Let $v$ be adjacent to $u$ and lying on the eccentric path connecting $u$ and $x_{i}$. All the vertices in the graph except $x_{i}$ are at distance atmost $n-1$ from $u$, where $n$ is the eccentricity of $u$. This implies $v$ being adjacent to $u$ will have eccentricity $n$. But $v$ lying on the symmetric circle can have eccentricity $\geq n+1$. Therefore the above graph cannot be an eccentric digraph.

Also we give a counter example for a problem given in [2] , as follows:

Problem 3, Ex. 2.2 (p. 41) [2]: If $G$ is self-centered with radius 2 , then $\bar{G}$ is self-centered with radius 2 .

Counter Example: Consider $C_{7}$, join the vertices at distance 2 in $C_{7}$. Let $G$ be the resulting graph with $\operatorname{rad}(G)=2$. Considering $\bar{G}$, we observe that $\bar{G} \cong C_{7}$; that is $\bar{G}$ is self-centered of radius 3 .

\section{Graphs with Isomorphic Eccentric Digraph}

In case of undirected graphs, Buckley [4] proved that the

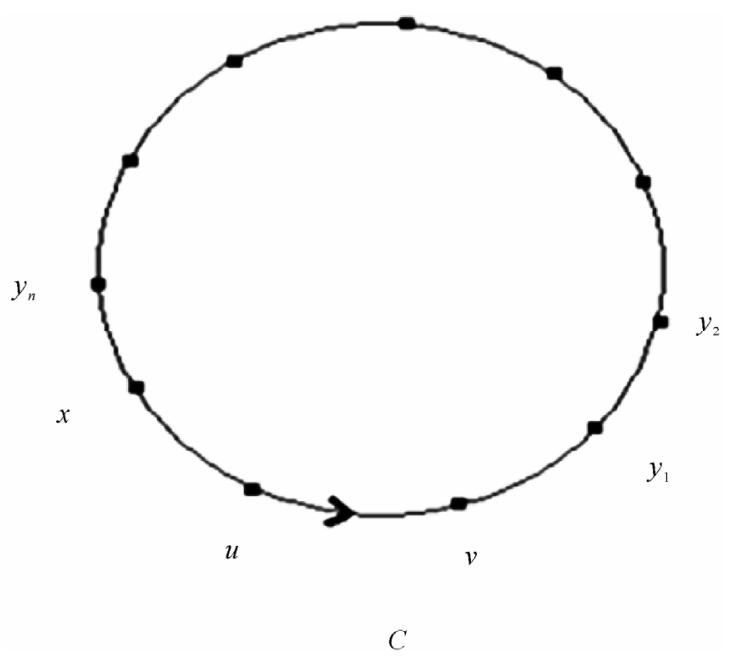

Figure 1. Directed cycle $C$.

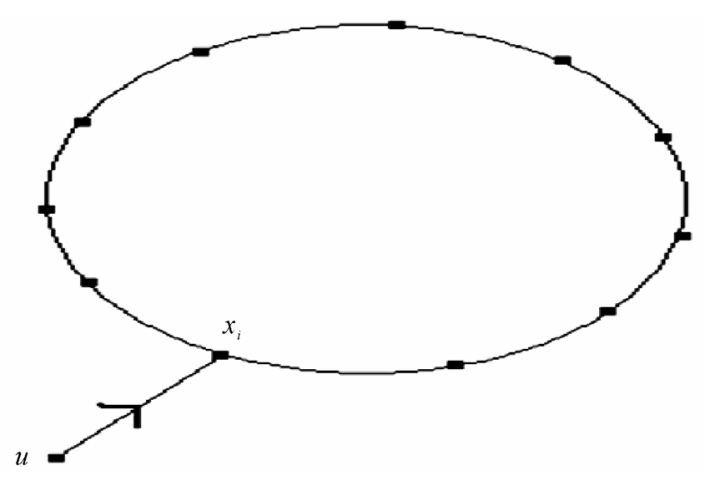

Figure 2. Directed cycle with unidirectional edge $u-x_{i}$. 
eccentric digraph of a graph $G$ is equal to its complement, $E D(G)=\bar{G}$, if and only if $G$ is either a self-centered graph of radius two or $G$ is the union of $k \geq 2$ complete graphs. In [9], Gimbert et al. have proved that the eccentric digraph $E D(G)$ is symmetric if and only if $G$ is self centered.

Here we are looking at graphs which have their eccentric digraphs isomorphic to themselves. So by Gimbert's result these graphs are self-centered graphs. In this section we consider self-centered, undirected graphs. The following observations are easily justified.

Remark 3. Odd cycles is a class of graphs for which $E D(G)=G$.

Remark 4. Odd cycles are graphs with minimum number of edges and maximum eccentricity on given number of vertices such that $E D(G)=G$.

Remark 5. For a self-centered graph $G$ with radius $\geq$ 3 , the complement $\bar{G}$ is self-centered with radius equal to two. Hence $G \subset \bar{G}$, and $G \nsubseteq \bar{G}$, and $E D(G)$ is isomorphic to a subgraph of $\bar{G}$. Further, by using Buckley's result [4], we can say that $E D(\bar{G})=\overline{\bar{G}}=G$. That is if $E D(\bar{G})=E D(G)$, then $G=E D(G)$.

Remark 6. Complete graphs is another class of graphs for which $E D(G)=G$.

Remark 7. It is easy to see that for graphs upto order 7, the only graphs for which $E D(G)=G$, are $K_{2}, K_{3}, K_{4}, K_{5}, C_{5}, K_{6}, K_{7}, C_{7}$.

Remark 8. Two isomorphic graphs have their eccentric digraphs isomorphic, but the converse need not be true always.

As an example, as shown inn Figure 3, we give a pair of non-isomorphic, self-centered graphs with same eccentricity having one eccentric digraph.

Lemma 9. Let $G$ be a self-centered graph with radius 2 , then $E D(G)=G$ if and only if $G$ is selfcomplementary.

Proof. Given self-centered graph $G$ be self-complementary with radius 2 . Then by Buckley's characterization theorem $[4], E D(G)=\bar{G}=G$. Conversley, consider a self-centered graph of radius 2, with $E D(G)=G$. Then, $E D(G)=\bar{G}$, that is $G=E D(G)=\bar{G}$. Hence the result.

Lemma 10. All self-centered graphs $G$ with eccentricity greater than or equal to 3 with $\bar{G}$ having period $=1$, tail $=1$, satisfies the condition $E D(G)=G$.

Proof. Let $G$ be a self-centered graph with eccentricity $\geq 3$. Then $\bar{G}$ is self-centered graph with eccentricity equal to 2 . Hence, $E D(\bar{G})=\overline{\bar{G}}=G$; that is $E D^{2}(\bar{G}) \cong E D(G)$. If $\bar{G}$ has period $=1$ and tail $=1$, that is $E D^{2}(\bar{G}) \cong E D(\bar{G})$ then $E D^{2}(\bar{G}) \cong E D(\bar{G}) \cong G$. But $E D^{2}(\bar{G}) \cong E D(G)$ implies

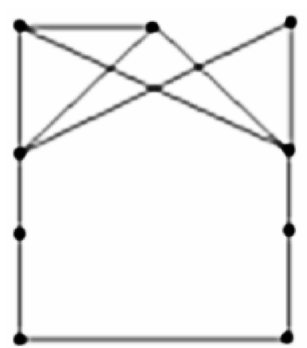

G

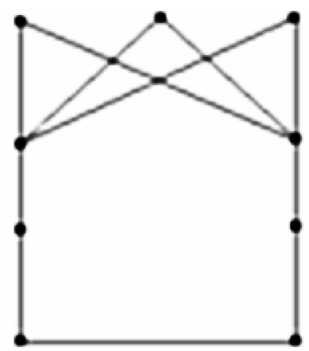

$H$

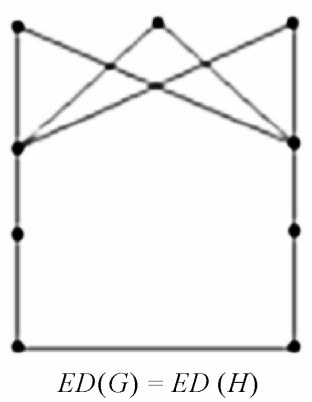

Figure 3. $E D(G)=E D(H)$.

$E D(G) \cong E D^{2}(\bar{G})=E D(\bar{G}) \cong G$. Hence the result.

For connected graph $G$ to be isomorphic to $E D(G)$ the necessary condition is that the graph should not be unique eccentric node graph as defined by Parthasarathy and Nandakumar [11]. Also, for $G=E D(G)$, the necessary condition is that the for every vertex of degree say $k$, there must exist another vertex with $k$ number of eccentric vertices. This can be defined as eccentric degree of a vertex.

Definition 11. For a vertex $v$ of a graph $G$ is defined to be the number of vertices at eccentric distance from $v$. Also the eccentric degree sequence of a graph is defined as a listing of eccentric degrees of vertices written in non-increasing order.

So for $E D(G)=G$, the eccentric degree sequence of $G$ should be equal to the degree sequence of $G$. But this condition is not sufficient as seen in the example below, depicted as Figure 4. Here both $G$ and $E D(G)$ have their degree seqence and eccentric degree sequences as $\left(3^{4}, 2^{9}\right)$, but $E D(G) \nsucceq G$.

Next, we consider self-centered graphs with given maximum degree $\Delta(G)$. By [2], $\Delta(G) \leq p-2 r+2$, for a self-centered graph $G$ with radius $r$. Our next result shows that there is no possibility of having a graph with $E D(G)=G$, with $\Delta(G)=p-2 r+2$.

Proposition 12. There does not exist a graph $G$ with $\Delta(G)=p-2 r+2$, such that $E D(G)=G$.

Proof. Let $G$ be a self-centered graph with $\Delta(G)=p-2 r+2$ Let $u \in V(G)$ such that $\operatorname{deg} u=p-2 r+2$. Partition the vertex set into sets lying 


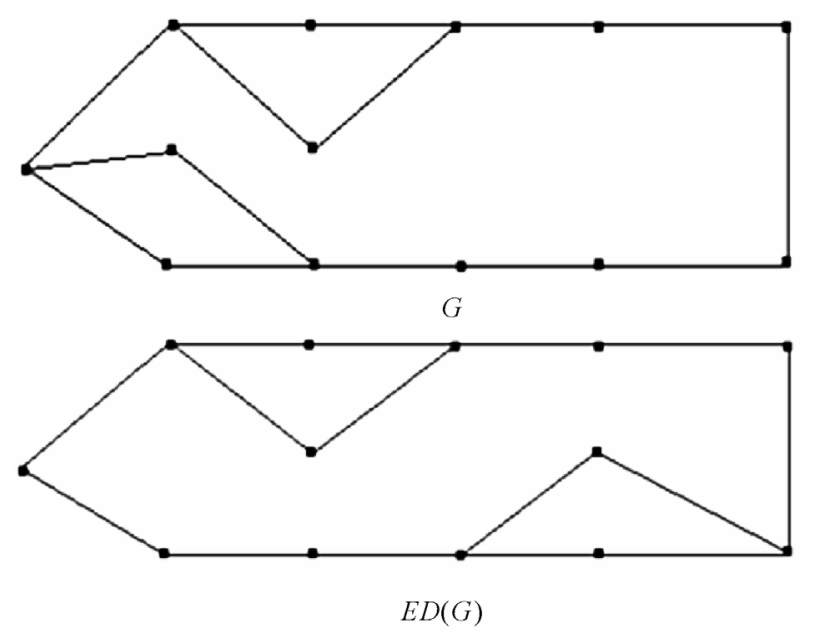

Figure 4. $G$ and $\operatorname{ED}(G)$ having same eccentric degree sequence and degree sequence, but $E D(G) \neq G$.

at distance $i$ from $u$ and name them as $A_{i}, 1 \leq i \leq r$. Since $\operatorname{deg} u=p-2 r+2,\left|A_{1}\right|=p-2 r+2$. As $G$ is self-centered, it cannot contain a cut-vertex and hence $\left|A_{i}\right| \geq 2,2 \leq i \leq r$. Hence, $2 r-2$ vertices are needed to satisfy the conditions of the graph under consideration, but we have $p-(p-2 r+2)=2 r-3$ vertices. Hence, it is not possible to construct a graph with $\operatorname{ED}(G)=G$, with $\Delta(G)=p-2 r+2$.

Theorem 13. A connected self-centered graph $G$ with $\Delta(G)=p-2 r+1$ is isomorphic to its eccentric digraph if and only if its degree sequence is of the form $(p-2 r+1)^{2}, 2^{p-2}$ with structure

$$
\begin{aligned}
& K_{1}+\overline{K_{p-2 r+1}} \cdot F \cdot \overline{K_{2}}+\left(\overline{K_{2}}-H\right)+\left(\overline{K_{2}}-H\right) \\
& +\cdots+\left(\overline{K_{2}}-H\right)\{r \text {-times }\}
\end{aligned}
$$

where $F$ is the graph obtained by joining one vertex of $\overline{K_{p-2 r+1}}$ to one vertex of $\overline{K_{2}}$ and remaining $p-2 r$ vertices to one vertex of $\overline{K_{2}}$, and $H$ is the 1 -factor removed from successive $\overline{K_{2}}+\overline{K_{2}}$.

Proof. Let $G$ be a self-centered graph with $\Delta(G)=p-2 r+1$. Let $u$ be a vertex of $G$ with $\operatorname{deg} u=p-2 r+1$. As seen in the above Lemma, each $A_{i}$ should contain at least two vertices each, for $G$ to satisfy $G=E D(G)$, with self-centeredness and not being unique eccentric node graph. Since the remaining $2 r-2$ vertices are to be distributed into $r-1$ sets with at least two in each set, it follows that each $A_{i}$ has exactly two vertices.

Let the vertices in the set $A_{k}$ be labelled $x_{k}^{1}$ and $x_{k}^{2}$ for all $k, 2 \leq k \leq r$. Since $G$ has no cutvertex, $x_{r-1}^{1}$ should be adjacent to at least one vertex of $A_{r}$, let us say, to $x_{r}^{1}$ and similarly let $x_{r-1}^{2}$ be adjacent to $x_{r}^{2}$. Degree of $x_{r}^{1}$ is at least two, so it can be adjacent to any of $x_{r}^{2}, x_{r-1}^{2}$ or both. Suppose, $x_{r}^{1}$ is adjacent to $x_{r-1}^{2}$, then $x_{r}^{1}$ will have $u$ as the only eccentric vertex, a contradiction.

Similar contradiction is arrived if $x_{r}^{1}$ is adjacent to both $x_{r}^{2}$ and $x_{r-1}^{2}$ Same argument can be applied in case of $x_{r}^{2}$. Therefore, $x_{r}^{1}$ and $x_{r}^{2}$ are mutually adjacent to have degree at least two. There are two paths $P_{1}$ and $P_{2}^{i j}$ where

$$
\begin{aligned}
P_{1}= & u, e_{1}^{i}, x_{1}^{i}, e_{2}^{j}, x_{2}^{1}, e_{3}^{1}, x_{3}^{1}, \cdots, e_{k-1}^{1}, x_{k}^{1}, e_{k+1}^{1}, x_{k}^{1}, \\
& e_{k+1}^{1}, x_{k+1}^{1}, \cdots, x_{r-2}^{1}, e_{r-1}^{1}, x_{r-1}^{1}, e_{r}^{1}, x_{r}^{1} ; i, j=1
\end{aligned}
$$

and

$$
\begin{aligned}
P_{2}^{i j}= & u, e_{1}^{i}, x_{1}^{i}, e_{2}^{j}, x_{2}^{2}, e_{3}^{2}, x_{3}^{2}, \cdots, \\
& e_{k-1}^{2}, x_{k-1}^{2}, e_{k}^{2}, x_{k}^{2}, e_{k+1}^{2}, x_{k+1}^{2}, \cdots, \\
& x_{r-2}^{2}, e_{r-1}^{2}, x_{r-1}^{2}, e_{r}^{2}, x_{r}^{2} ; 2 \leq i, j \leq p-2 r+1,
\end{aligned}
$$

$i$ need not be equal to $j$.

Other cases are proved as follows:

Claim 1: The vertices $x_{k+1}^{1}$ are adjacent to either $x_{k}^{1}$ or $x_{k}^{2}$, but not both; for all $k, 2 \leq k \leq r-1$.

Proof of the claim: Since $G$ has no cut vertex each vertex belonging to $A_{k}, 2 \leq k \leq r-1$, is adjacent to at least one vertex in $A_{k-1}$ and $A_{k+1}$. Without loss generality let each vertex $x_{k+1}^{1}$ be adjacent to $x_{k}^{1}$, for all $k, 2 \leq k \leq r-1$. Let us consider $A_{k}$, with vertices $x_{k}^{1}$ and $x_{k}^{2}$. Let $x_{k+1}^{1}$ be adjacent to $x_{k}^{2}$ along with $x_{k}^{1}$. Then eccentricity of $x_{k+1}^{1}$ can be at most $r-1$ as any vertex lying on the path $P_{1}$ or $P_{2}^{i j}$ can be at most at a distance $r-1$ from $x_{k+1}^{1}$. And any vertex on the path $P_{2}^{i j} \quad$ can be at most at a distance of $r-2$ from $x_{k}^{2}$. In any case if $x_{k+1}^{1}$ is adjacent to both $x_{k}^{2}$ and $x_{k}^{1}$ then $\mathrm{G}$ ceases to be self-centered, a contradiction and hence the proof of the claim.

Claim 2: Each $A_{i}, 2 \leq k \leq r-1$, is independent, that is, $\left\langle A_{i}\right\rangle=\overline{K_{2}}$.

Proof of the claim: First we prove the result for $A_{r-1}$. If the vertices $x_{r-1}^{1}$ and $x_{r-1}^{2}$ belonging to $A_{r-1}$ are adjacent then $x_{r-1}^{1}$ will have $u$ as the only eccentric vertex, a contradiction proves that $\left\langle A_{r-1}\right\rangle=\overline{K_{2}}$.

For any other $A_{k}, 2 \leq k \leq r-2$, if $x_{k}^{1}$ and $x_{k}^{2}$ are adjacent then, eccentricity of $x_{k}^{1}$ and $x_{k}^{2}$ will be at most $r-2$ as vertices on $P_{1}$ or $P_{2}^{i j}$ will be at distance at most $r-2$ from $x_{k}^{1}$ or $x_{k}^{2}$ and hence for all $k$, $2 \leq k \leq r-1,\left\langle A_{i}\right\rangle=\bar{K}_{2}$.

Claim 3: $x_{2}^{1}$ and $x_{2}^{2}$ do not have common neighbors in $A_{1}$.

Proof of the claim: As in case of the vertices of $A_{r-1}$, the vertices $x_{2}^{1}$ and $x_{2}^{2}$ of $A_{2}$ will have their eccentricity equal to $r-1$ if they have a common neighbor in $A_{1}$, hence the claim.

Claim 4: $x_{2}^{1}$ is adjacent to $x_{1}^{1}$ and $x_{2}^{2}$ is adjacent to all other $\mathrm{p}-2 \mathrm{r}$ vertices of $A_{1}$.

Proof of the claim: If $x_{2}^{1}$ is adjacent to 
$\left\{x_{1}^{k}\right\}_{k=1}^{k=i}, 1<i<p-2 r$, then two vertices $x_{r}^{2}$ and $x_{r-1}^{2}$

have eccentric degree $k+1$, but only one vertex $x_{2}^{1}$ has degree equal to $k+1$, a contradiction.

If $x_{2}^{1}$ and $x_{2}^{2}$ have degree $k$ each ,that is if $2 k=p-2 r+1$, then $x_{r}^{1}, x_{r}^{2}, x_{r-1}^{1}$ and $x_{r-1}^{2}$ have eccentric degree equal to $k+1$, but only two vertices $x_{2}^{1}$ and $x_{2}^{2}$ have degree $k+1$.

Hence, $x_{2}^{1}$ is adjacent to $x_{1}^{1}$ and $x_{2}^{1}$ not adjacent to $x_{1}^{i}, 1<i<p-2 r$.

Finding the eccentric degree of all vertices of $G$ we see that, ecc. $\operatorname{deg}\left(x_{k}^{1}\right)=2,1 \leq k \leq r-1$ and ecc.deg $\left(y_{k}^{1}\right)=2$, except for $x_{r-1}^{1}$, where $y_{k}^{1} \in N\left(x_{k}^{1}\right)$.

Similarly, ecc. $\operatorname{deg}\left(x_{k}^{2}\right)=2,1 \leq k \leq r-1$ and ecc.deg $\left(y_{k}^{2}\right)=2$, where $y_{k}^{2} \in N\left(x_{k}^{2}\right)$. Hence, the eccentric degree sequence of $G$ is $(p-2 r+1)^{2}, 2^{p-2}$, which is same as that of degree sequence of $G$.

Claim 5: $\left\langle A_{1}\right\rangle=\overline{K_{p-2 r+1}}$.

Proof of the claim: We show that for any two vertices $x_{1}^{t}$ and $x_{1}^{s}$ where $t \neq s, 1 \leq t, s \leq p-2 r+1$, are not adjacent. Here we need to consider two possibilities:

Case 1): $x_{1}^{t} \in N\left(x_{2}^{1}\right)$ and $x_{1}^{s} \in N\left(x_{2}^{2}\right)$

Case 2): $x_{1}^{t} \in N\left(x_{2}^{2}\right)$ and $x_{1}^{s} \in N\left(x_{2}^{2}\right)$

In case 1$), x_{1}^{t}$ will have only one eccentric vertex, a contradiction.

In case 2$), \operatorname{deg}\left(x_{1}^{t}\right), \operatorname{deg}\left(x_{1}^{s}\right) \geq 3$ then only the vertices $x_{r}^{1}$ or $x_{r}^{2}$ of $A_{r}$ and $x_{r-1}^{1}$ or $x_{r-1}^{2}$ of $A_{r-1}$ can have vertices of $A_{1}$ as eccentric vertices, along the paths $P_{1}$ or $P_{2}^{i j}$, since $x_{2}^{1}$ and $x_{2}^{2}$ do not share a common neighbor in $A_{1}$, as claimed in 3 .

By Claim 4, we see that $x_{2}^{1}$ is adjacent to $x_{1}^{1}$ and $x_{2}^{2}$ is adjacent to all other $p-2 r$ vertices of $A_{1}$, implies that when degrees of $x_{1}^{t}$ and $x_{1}^{s}$ are changed by making them adjacent, the eccentric degree of any other vertex of does not change, hence we will not be able to get $E D(G)=G$, a contradiction proves the claim.

Referring all the claims we conclude that $G$ is of the form defined in the statement of the theorem.

Converse is easy to observe that if $G$ is as given in the statement of the theorem, then $\operatorname{ED}(G)=G$.

In the next result we consider a particular case of graphs with $E D(G)=G$, that is, odd cycles.

Remark 14. In a labelled $C_{2 n+1}, n \geq 1$, two vertices $v_{i}, v_{j}$ are at eccentric distance in $\operatorname{ED}\left(C_{2 n+1}\right)$, if and only if $d_{G}\left(v_{i}, v_{j}\right)=\frac{n}{2}$ or $\frac{n+1}{2}$.

Remark 15. For unlabelled odd cycles, iterations of $\operatorname{ED}\left(C_{2 n+1}\right)$ can be packed into $K_{n}$, since there are $\frac{n-1}{2}-1=\frac{n-3}{2}, \operatorname{ED}(G)^{\prime} s$, whereas, $\operatorname{rad}\left(C_{2 n+1}\right)=n$.

In case of labelled odd cycles the sequence of $\operatorname{ED}(G)$ 's can be packed into $K_{p}$, if the permutation on $\mathrm{p}$ number of vertices defined by

$$
\begin{aligned}
& f(1)=1, f(2 i)=r+2-i, i=1,2,3, \cdots, r \\
& f(2 i+1)=2 r+2-i, i=1,2,3, \cdots, r
\end{aligned}
$$

is a product of three cyclic permutations of length $1, r, r$, respectively.

Proposition 16. There exists a self-centered graph $G$, such that $\operatorname{ED}(G)=G$, containing an odd cycle.

Proof. Let be $C_{p}$ be a cycle, whose vertices are labeled as $1,2,3, \cdots, p$. Let $S=\{1,2,3, \cdots, p\}$. Define a function on the set of vertices of $C_{p}$ as

$$
\begin{aligned}
& f(1)=1, f(2 i)=((p-2 i+3) / 2), 1 \leq i \leq((p-1) / 2), \\
& f(2 i+1)=p+1-i, 1 \leq i \leq((p-1) / 2) .
\end{aligned}
$$

Now, we partition of set of vertices of $C_{p}$ into $\left\{S_{1}, S_{2}, S_{3}, \cdots, S_{m}\right\}$ where,

$$
S_{1}=(1), S_{2}=\left\{2, f(2), f^{2}(2), \cdots, f^{n}(2)\right\}, 2 \notin S_{1},
$$

where $n$ is the least positive integer such that $f^{n+1}(2)=2$ whereas $f^{n}(2)$ is obtained by applying $f$ on $2, n$ times. Similarly,

$$
\begin{aligned}
& S_{m}=\left\{l, f(l), f^{2}(l), \cdots, f^{m-1}(l)\right\}, \\
& l \notin S_{1} \cup S_{2} \cup S_{3} \cup \cdots \cup S_{m-1},
\end{aligned}
$$

where $m$ is the least positive integer such that $f^{m+1}(l)=l$. It is clear that $S_{1} \cup S_{2} \cup S_{3} \cup \cdots \cup S_{m}=S$ and $S_{i} \cap S_{j}=\phi, 1 \leq i, j \leq m, i \neq j$. Now, for each vertex of $S_{i}$ we define sets of vertices not in $C_{p}$ by $S_{i_{j}}=\left\{f_{i_{j}}(l), f_{i_{j}}^{2}(l), \cdots, f_{i_{j}}^{n_{i}}(l)\right\}$ for each $i$, and $j=1,2,3, \cdots$ whose adjacencies are to the vertices of $C_{p}$ defined by: If $f^{g}(l)$, when $1 \leq l \leq p$ is adjacent to $f^{h}(l)$, then, $f_{i_{j}}^{g}(l)$ is adjacent to $N\left(f^{g}(l)\right)$, $f_{i_{j}}^{h}(l)$ and $f_{i_{j}}^{h}(l)$ is adjacent to $N\left(f^{h}(l)\right), f_{i_{j}}^{g}(l)$; otherwise, $f_{i_{j}}^{g}(l)$ is adjacent to $N\left(f^{g}(l)\right)$ and $f_{i_{j}}^{h}(l)$ is adjacent to $N\left(f^{h}(l)\right)$. So we get a self-centered graph with radius $(p-1) / 2$, satisfying $E D(G)=G$, as vertices $l$ on $C_{p}$ and respective $f_{i_{j}}^{m}(l), 1 \leq l \leq p$, have same distance from other vertices in the graph. By Remark 15, we get $E D(G) \cong G$.

Following is an example of a self-centered graph with radius 4, as shown in Figure 5, satisfying $E D(G) \cong G$, with $C_{9}$, as base. So $S=\{1,2, \cdots, 9\}$ and $V\left(C_{p}\right)=\left\{S_{1}, S_{2}, S_{3}, S_{4}, S_{5}\right\}$, where $S_{1}=\{1\}, S_{2}=\{2,5,8\}, S_{3}=\{4\}, S_{4}=\{7\}, S_{5}=\{3,6,9\}$. 


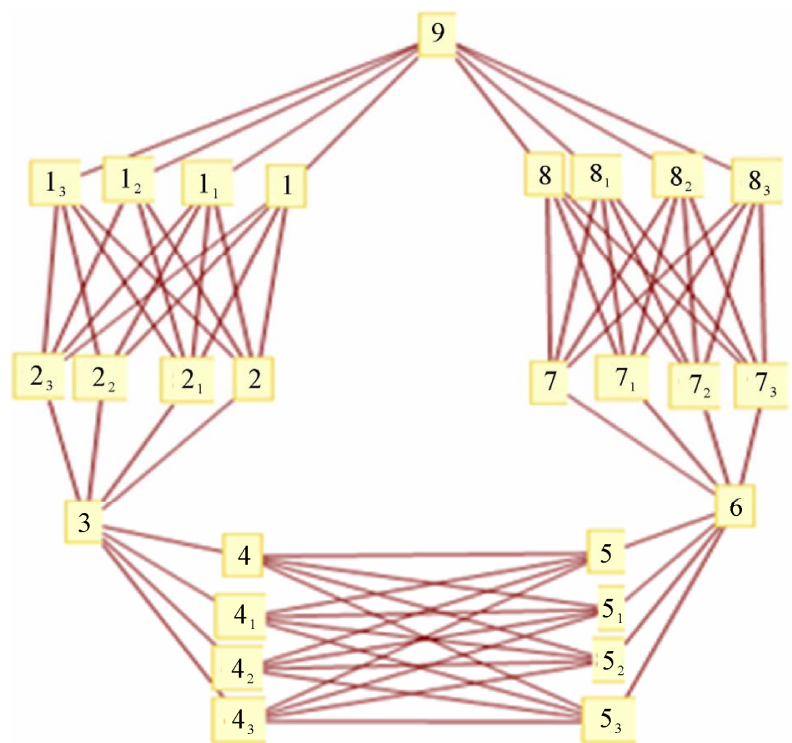

Figure 5. $E D(G) \cong G$.

For $S_{i}, 1 \leq i \leq 4$, we have

$S_{1_{1}}=\left\{1_{1}\right\}, \quad S_{1_{2}}=\left\{1_{2}\right\}, S_{1_{3}}=\left\{1_{3}\right\} ; S_{2_{1}}=\left\{2_{1}, 5_{1}, 8_{1}\right\}$,

$S_{2_{2}}=\left\{2_{2}, 5_{2}, 8_{2}\right\}, S_{2_{3}}=\left\{2_{3}, 5_{3}, 8_{3}\right\} ; S_{3_{1}}=\left\{4_{1}\right\}$,

$S_{3_{2}}=\left\{4_{2}\right\}, S_{3_{3}}=\left\{4_{3}\right\} ; S_{4_{1}}=\left\{7_{1}\right\}, S_{4_{2}}=\left\{7_{2}\right\}, S_{4_{3}}=\left\{7_{3}\right\}$.

\section{References}

[1] M. I. Huilgol, S. A. S. Ulla and A. R. Sunilchandra, "On Eccentric Digraphs of Graphs," Proceedings of the International Conference on Emerging Trends in Mathematics and Computer Applications, MEPCO Schlenk En- gineering College, Sivakasi, 16-18 December 2010, pp. 41-44.

[2] F. Buckley and F. Harary, "Distance in Graphs," Addison-Wesley, Redwood City, 1990.

[3] G. Chartrand and L. Lesniak, "Graphs and Digraphs," 3rd Edition, Chapman \& Hall, London, 1996.

[4] F. Buckley, "The Eccentric Digraph of a Graph," Congressus Numerantium, Vol. 149, 2001, pp. 65-76.

[5] G. Johns and K. Sleno, "Antipodal Graphs and Digraphs," International Journal of Mathematics and Mathematical Sciences, Vol. 16, No. 3, 1993, pp. 579-586. doi:10.1155/S0161171293000717

[6] R. Aravamudhan and B. Rajendran, "On Antipodal Graphs," Discrete Mathematics, Vol. 49, No. 1, 1984, pp. 193-195. doi:10.1016/0012-365X(84)90117-1

[7] J. Akiyama, K. Ando and D. Avis, "Eccentric Graphs," Discrete Mathematics, Vol. 56, No. 1, 1985, pp. 1-6. doi:10.1016/0012-365X(85)90188-8

[8] J. Boland and M. Miller, "The Eccentric Digraph of a Digraph," Proceedings of the 12th Australasian Workshop of Combinatorial Algorithms (AWOCA 2001), Freiburg, 3-7 September 2001, pp. 66-70.

[9] J. Gimbert, M. Miller, F. Ruskey and J. Ryan, "Iterations of Eccentric Digraphs," Bulletin of the Institute of Combinatorics and Its Applications, Vol. 45, 2005, pp. 41-50.

[10] J. Boland, F. Buckley and M. Miller, "Eccentric Digraphs," Discrete Mathematics, Vol. 286, No. 1-2, 2004, pp. 25-29. doi:10.1016/j.disc.2003.11.041

[11] R. Nandakumar and K. R. Pathasarathy, "Unique Eccentric Point Graphs," Discrete Mathematics, Vol. 46, No. 1, 1983, pp. 69-74. doi:10.1016/0012-365X(83)90271-6 